\title{
Metabolic Alteration in Cancer Cells by Therapeutic Carbon Ions
}

\author{
NAOTO OSU ${ }^{1}$, HIDEKI MAKINOSHIMA ${ }^{2,3,4}$, TAKAHIRO OIKE ${ }^{1,5}$ and TATSUYA OHNO ${ }^{1,5}$ \\ ${ }^{1}$ Department of Radiation Oncology, Gunma University Graduate School of Medicine, Maebashi, Japan; \\ ${ }^{2}$ Shonai Regional Industry Promotion Center, Tsuruoka, Japan; \\ ${ }^{3}$ Tsuruoka Metabolomics Laboratory, National Cancer Center, Tsuruoka, Japan; \\ ${ }^{4}$ Division of Translational Research, Exploratory Oncology Research, and \\ Clinical Trial Center, National Cancer Center, Kashiwa, Japan; \\ ${ }^{5}$ Gunma University Heavy Ion Medical Center, Maebashi, Japan
}

\begin{abstract}
Background/Aim: Carbon-ion radiotherapy has strong antitumor effects in X-ray-resistant tumors. However, the mechanisms underlying the strong antitumor effect, especially on metabolic alterations, are not fully elucidated. This study aimed to determine the effect of therapeutic carbon ions on metabolic alterations in cancer cells. Materials and Methods: Five human cancer cell lines were used in this study. The change in 333 metabolite levels in response to carbon ions was analyzed using gas chromatography-mass spectrometry. Results: Fifty-two metabolites were commonly detected in all cell lines. The levels of five metabolites significantly changed in four or more cell lines. Three of the five metabolites (i.e., 2ketoglutaric acid, fumaric acid and 2-hydroxyglutaric acid) were associated with the TCA cycle. TCA cycle intermediates and the downstream oncometabolite 2-hydroxyglutaric acid were up-regulated by carbon ions. Conclusion: We demonstrated for the first time that TCA cycle intermediates and 2-hydroxyglutaric acid are up-regulated after carbonion irradiation.
\end{abstract}

Carbon ion radiotherapy is a promising cancer treatment that has a strong antitumor effect in tumors resistant to X-ray radiotherapy (1). The mechanisms underlying the strong

This article is freely accessible online.

Correspondence to: Takahiro Oike, MD, Ph.D., Associate Professor, Department of Radiation Oncology, Gunma University Graduate School of Medicine, 3-39-22, Showa-machi, Maebashi, Gunma 371-8511, Japan. Tel: +81 272208383, Fax: +81 272208397, e-mail: oiketakahiro@gunma-u.ac.jp

Key Words: Metabolomics, carbon ions, radiotherapy, cancer cell lines. antitumor effect have been studied mainly in terms of the DNA damage response (2). Carbon ions induce larger and more complex DNA double-strand breaks than X-rays (3) and efficiently induce a mode of cell death called mitotic catastrophe (4). However, other than DNA damage, the biological responses of cancer cells to carbon ions have not been fully elucidated.

Metabolomics is the comprehensive analysis of small molecular metabolites in an organism (5). In recent years, metabolomics has attracted much attention in cancer research because of the development of analytical technologies based on chromatography and mass spectrometry. Previous studies have identified metabolic alterations characteristic to cancer cells, such as the Warburg effect (6), and cancer cell-specific increases in metabolites such as 2-hydroxyglutaric acid (2HG), fumaric acid, and creatine riboside $(7,8)$. These metabolic alterations and metabolites characteristic of cancers are promising therapeutic targets or biomarkers for cancer. However, the metabolic alterations induced by carbon ions in cancer cells have not been fully elucidated. To address this issue, we comprehensively analyzed the changes in metabolite levels in response to therapeutic carbon ion beams in several human cancer cell lines of various origins using gas chromatography coupled to mass spectrometry (GC-MS).

\section{Materials and Methods}

Cell lines and cell culture. Human cancer cell lines (A549, MIA PaCa-2, MeWo, HuCCa-1 and U2OS cells) were used in this study (Table I). The cells were cultured in RPMI-1640 (Sigma-Aldrich, St. Louis, MO, USA) supplemented with $10 \%$ fetal bovine serum (Life Technologies, Carlsbad, CA, USA) at $37^{\circ} \mathrm{C}$ with $5 \% \mathrm{CO}_{2}$.

Irradiation. Carbon ion irradiation was performed at Gunma University Heavy Ion Medical Center. The specific parameters were as follows: an energy of $290 \mathrm{MeV} /$ nucleon and average linear 
Table I. Cell lines used in this study.

\begin{tabular}{lcc}
\hline Cell line name & Origin & Purchased from \\
\hline A549 & Lung & $\mathrm{a}$ \\
MIA PaCa-2 & Pancreas & $\mathrm{b}$ \\
MeWo & Skin & $\mathrm{a}$ \\
HuCCa-1 & Intrahepatic bile duct & $\mathrm{b}$ \\
U2OS & Bone & $\mathrm{a}$ \\
\hline
\end{tabular}

a, ATCC (Manassas, VA, USA); b, JCRB (Ibaraki, Japan).

energy transfer (LET) at the center of a $6 \mathrm{~cm}$ spread-out Bragg peak (SOBP) of approximately $50 \mathrm{keV} / \mu \mathrm{m}$. X-ray irradiation was performed using an MX-160 Labo (160 kVp, $1.06 \mathrm{~Gy} / \mathrm{min}$; mediXtec, Matsudo, Japan) (9). $1.0 \times 10^{6}$ cells were seeded on $10 \mathrm{~cm}$ dishes at a density that equates to $1-5 \times 10^{6}$ cells at the time of collection, incubated at $37^{\circ} \mathrm{C}$ overnight, and then irradiated (or sham-irradiated) with $1 \mathrm{~Gy}$ of carbon ions or $3 \mathrm{~Gy}$ of X-rays.

Sample processing and GC-MS. Twenty-four hours after irradiation, culture dishes were washed twice with 5\% mannitol (Wako, Osaka, Japan). Then, $800 \mu$ methanol (Wako) was added to the culture dish, which was incubated at room temperature for 1 min. $10 \mu \mathrm{l}$ of 0.5 $\mu \mathrm{g} / \mu \mathrm{l}$ of isopropyl malic acid (Sigma-Aldrich) was added to the dish as an internal standard. The metabolite extract was transferred into a microfuge tube and dried using a spin dryer (TAITEC, Koshigaya, Japan). Derivatization in the solid phase was performed as previously described (10). Derivatized samples were eluted with $100 \mu \mathrm{l}$ of nhexane and $1.0 \mu \mathrm{l}$ of the derivatized solution was injected into a GCMS machine (GCMS-TQ8050, Shimadzu, Kyoto, Japan). Analysis of the metabolome was performed as described previously (11). Metabolite detection was performed using the Smart Metabolites Database and GCMS Solution software (Shimadzu, Kyoto, Japan) (11). The area under the curve for each metabolite was normalized to that of the internal standard and defined as the metabolite level after normalization to the cell number.

Metabolomics data analysis and statistics. Principal component analysis (PCA) was performed using MetaboAnalyst 5.0 (12). The elliptical 95\% confidence region was calculated as the region where $95 \%$ of the metabolites detected in the given experimental conditions were included in the scatter plot using two axes, PC1 and PC2 determined by Metaboanalyst 5.0. Metabolomic mapping was performed using VANTED (13). Differences between two groups were examined using the Student's $t$-test $(14,15)$ with the Benjamini-Hochberg procedure (16). Statistical significance was set at $p \leq 0.05$. All experiments were performed in triplicate.

\section{Results}

To elucidate the metabolic alterations induced by carbon ions in cancer cells, five human cancer cell lines originating from various cancer types were treated with carbon ion radiotherapy, and 333 metabolites were comprehensively analyzed using GC-MS (Supplementary Data). The results showed that 123 metabolites were detected in A549 cells,

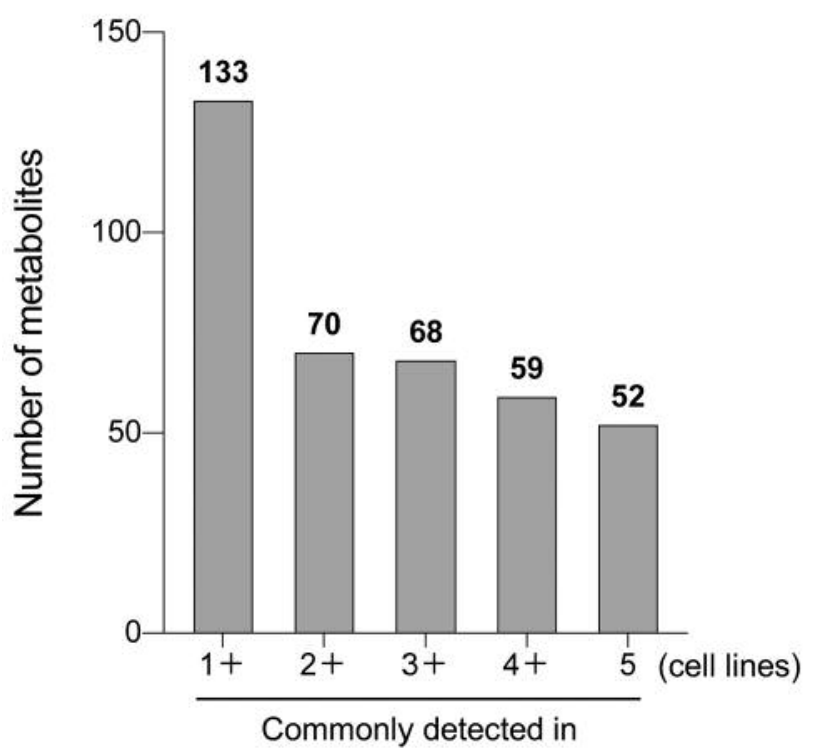

Figure 1. The number of metabolites detected in one or more types of human cancer cell lines by GC-MS analysis.

100 metabolites in MIA PaCa- 2 cells, 75 metabolites in MeWo cells, 106 metabolites in HuCCa-1 cells and 109 metabolites in U2OS cells (Figure 1); 52 metabolites were commonly detected in all cell lines (Supplementary Table I).

To confirm the robustness of the GC-MS analysis, PCA was performed on the 52 metabolites detected in all cell lines. A distinct separation between the control group and the carbon ion-treated group was observed in all cell lines (Figure 2). This result indicates that the GC-MS analysis used in this study is robust.

Next, the changes in the levels of metabolites induced by carbon ions were evaluated for each cell line (Supplementary Tables II-VI). The results showed that levels of 2-HG, 3hydroxyglutaric acid, and fumaric acid significantly changed in all cell lines (Table II). The levels of 2-ketoglutaric acid $(\alpha-K G)$ and niacinamide significantly changed in four cell lines (Table II). The levels of these five metabolites increased in response to carbon ion irradiation (Figure 3).

$\alpha-K G$ and fumaric acid are intermediary metabolites of the tricarboxylic acid (TCA) cycle. 2-HG is an oncometabolite derived from $\alpha-K G$ (17). Therefore, three of the five metabolites that significantly increased are associated with the TCA cycle. Plotting details of these metabolites on a metabolic map demonstrated that carbon ions up-regulated the TCA cycle and 2-HG levels in all cell lines (Figure 4). Interestingly, $1 \mathrm{~Gy}$ of carbon ions tended to up-regulate the TCA cycle and 2-HG levels to a greater level than $3 \mathrm{~Gy}$ of $\mathrm{X}$-rays. Together, these data indicate that carbon ion irradiation of cancer cells induces metabolic alterations, characterized by up-regulation of TCA cycle intermediates. 
A

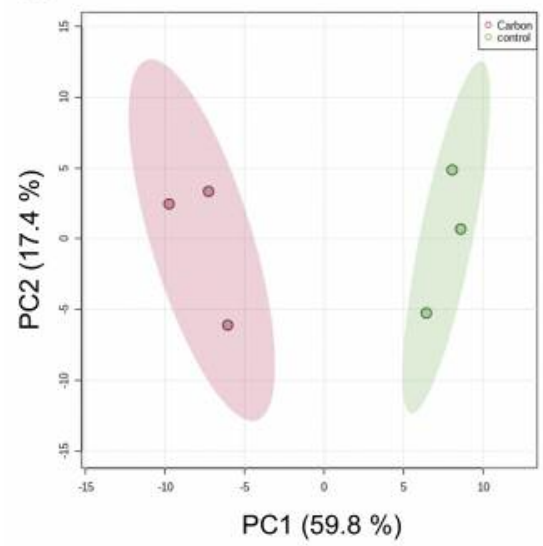

D

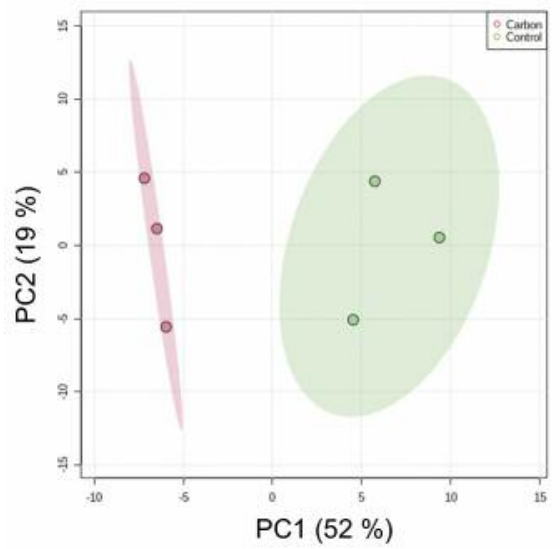

B

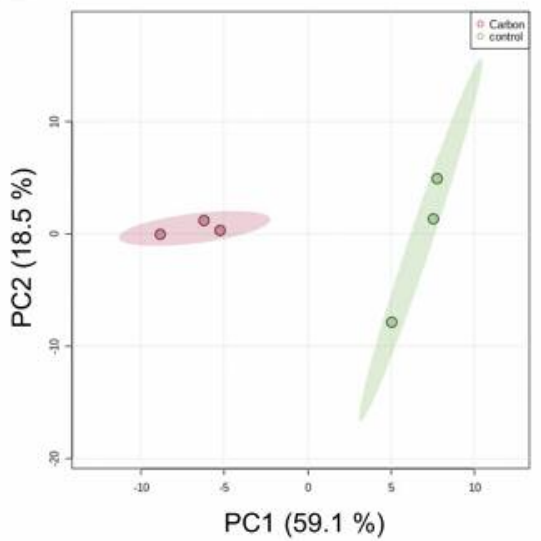

E

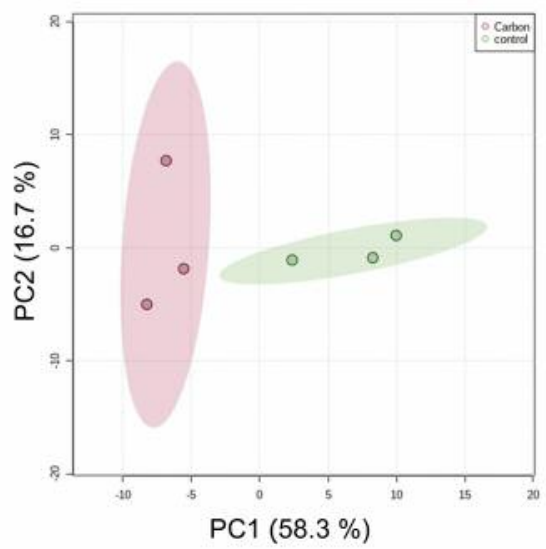

C
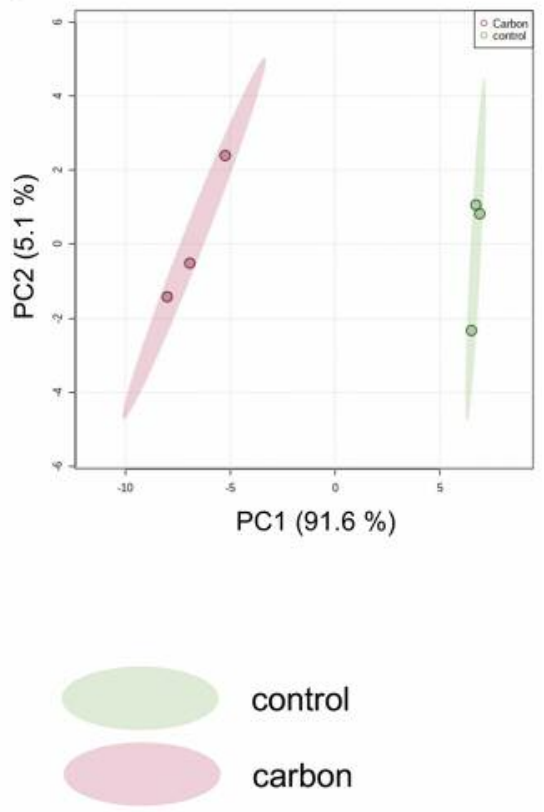

Figure 2. Principal component analysis (PCA) score plots for discriminating the control group and the carbon ion-treated group in each cell line. The number in parentheses on the PC1 and PC2 axes shows the contribution rate. The colored elliptical area shows a 95\% confidence region for control (green) and carbon ions (red). (A) A549, (B) MIA PaCa-2, (C) MeWo, (D) HuCCa-1, and (E) U2OS cells. PC, Principal component.

Table II. Metabolites that significantly changed in four or more cell lines after carbon ion irradiation.

\begin{tabular}{|c|c|c|c|c|c|c|c|c|c|c|c|c|c|c|c|}
\hline \multirow{2}{*}{$\begin{array}{l}\text { Cell line } \\
\text { Metabolite }\end{array}$} & \multicolumn{3}{|c|}{ A549 } & \multicolumn{3}{|c|}{ MIA PaCa-2 } & \multicolumn{3}{|c|}{ MeWo } & \multicolumn{3}{|c|}{$\mathrm{HuCCa}-1$} & \multicolumn{3}{|c|}{ U2OS } \\
\hline & $\mathrm{FC}$ & $p$-Value & $q$-Value & $\mathrm{FC}$ & $p$-Value & $q$-Value & $\mathrm{FC}$ & $p$-Value & $q$-Value & $\mathrm{FC}$ & $p$-Value & $q$-Value & $\mathrm{FC}$ & $p$-Value & $q$-Value \\
\hline 2-HG & 1.7 & 0.002 & 0.020 & 1.6 & $7.1 \mathrm{E}-05$ & 0.002 & 2.2 & 0.008 & 0.013 & 1.3 & 0.002 & 0.021 & 1.4 & 0.002 & 0.015 \\
\hline 3-HG & 1.5 & $1.3 \mathrm{E}-05$ & $6.6 \mathrm{E}-04$ & 1.7 & $7.9 \mathrm{E}-07$ & $4.1 \mathrm{E}-05$ & 2.9 & $1.3 \mathrm{E}-06$ & $6.9 \mathrm{E}-05$ & 1.3 & $8.2 \mathrm{E}-05$ & 0.004 & 1.3 & $2.5 \mathrm{E}-04$ & 0.013 \\
\hline Fumaric acid & 1.7 & 0.003 & 0.024 & 1.4 & $2.1 \mathrm{E}-04$ & 0.003 & 2.5 & 2.3E-04 & 0.002 & 1.3 & 0.001 & 0.019 & 1.3 & 0.009 & 0.035 \\
\hline$\alpha-K G$ & 1.6 & 4.1E-04 & 0.007 & 1.5 & $1.4 \mathrm{E}-04$ & 0.002 & 2.5 & 7.9E-05 & 0.001 & 1.5 & 0.033 & 0.071 & 1.4 & 0.010 & 0.034 \\
\hline Niacinamide & 1.6 & 0.001 & 0.014 & 1.8 & 0.022 & 0.062 & 2.3 & 0.003 & 0.006 & 1.6 & 0.007 & 0.032 & 1.4 & 0.002 & 0.013 \\
\hline
\end{tabular}

2-HG, 2-Hydroxyglutaric acid; 3-HG, 3-hydroxyglutaric acid; $\alpha$-KG, 2-ketoglutaric acid; FC, fold change. $p$-Values assessed by Student's $t$-test and $q$-values, that are the $p$-Values adjusted by Benjamini-Hochberg procedure, are shown.

\section{Discussion}

This study revealed metabolic alterations in cancer cells induced by carbon ions using GC-MS. To the best of our knowledge, no previous studies have demonstrated that carbon ion irradiation up-regulates the TCA cycle in cancer cells; there are several reports on the effects of X-ray irradiation on the TCA cycle in non-cancerous samples. Goudarzi et al. 


\section{2-hydroxyglutaric acid}

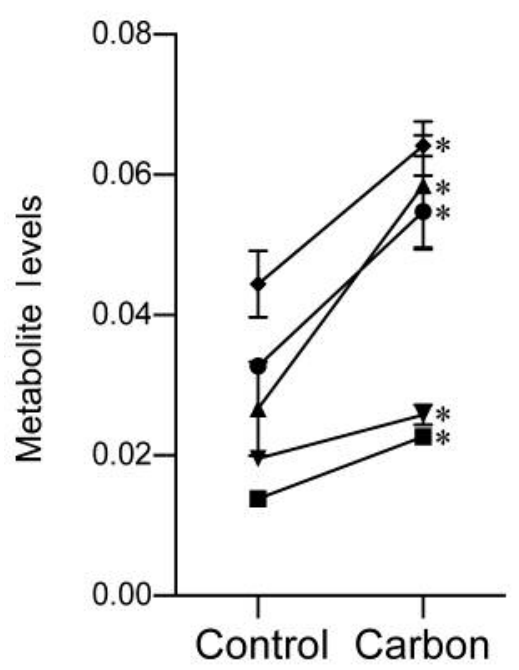

2-ketoglutaric acid

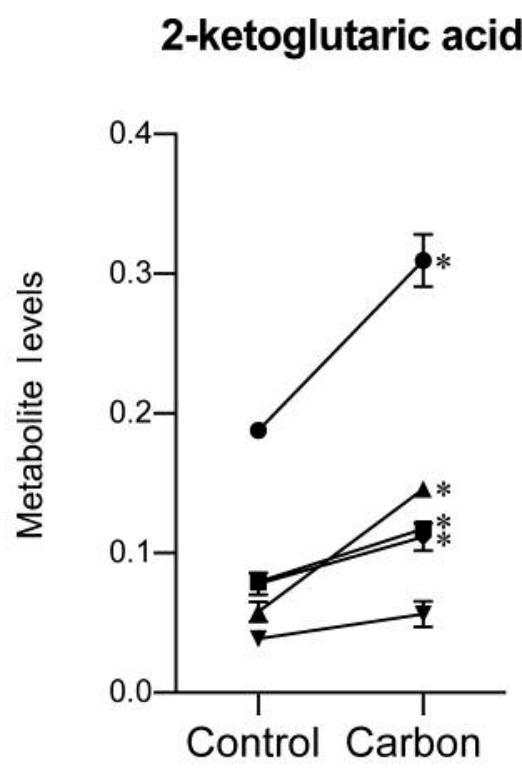

3-hydroxyglutaric acid
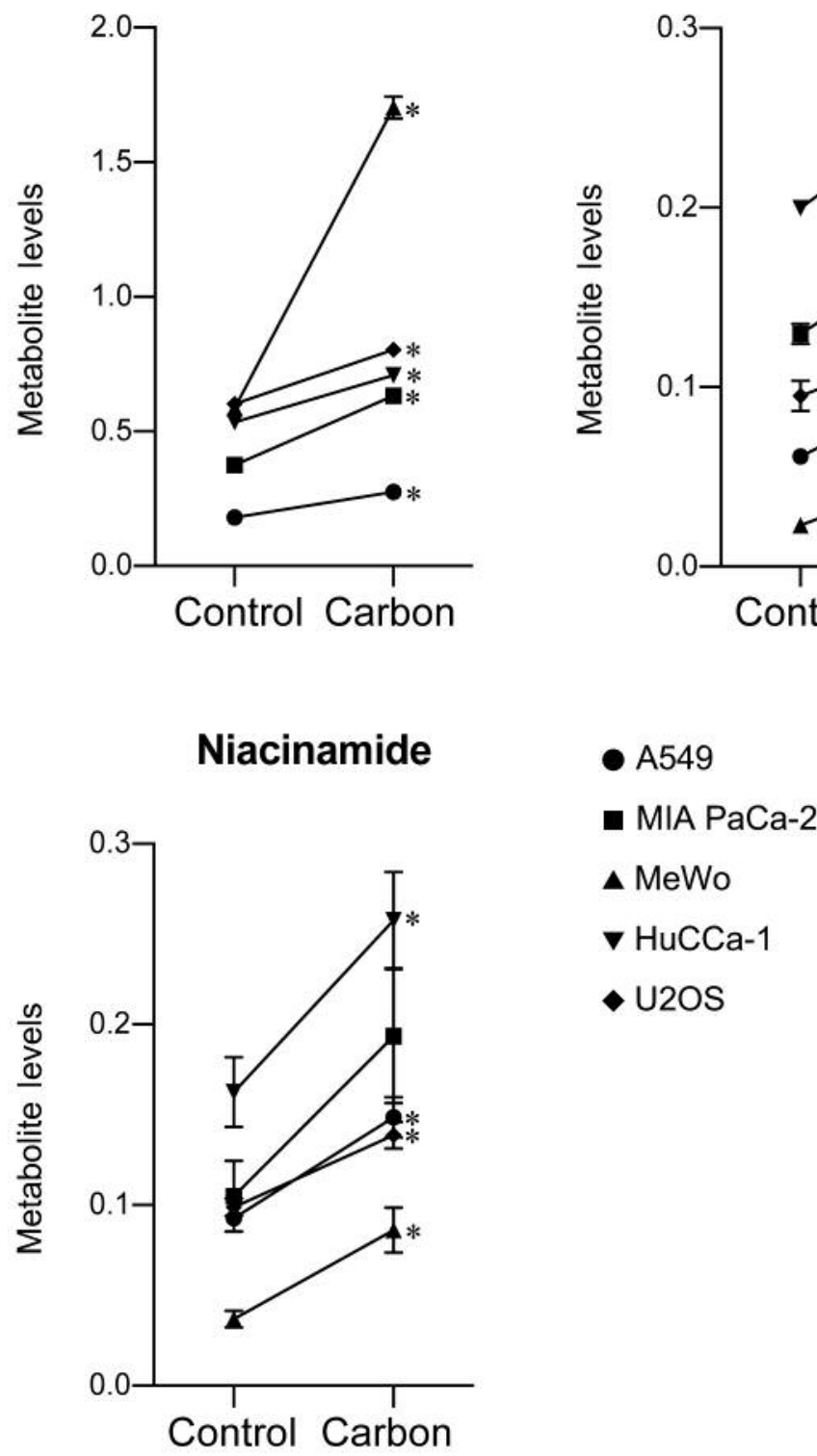

Fumaric acid

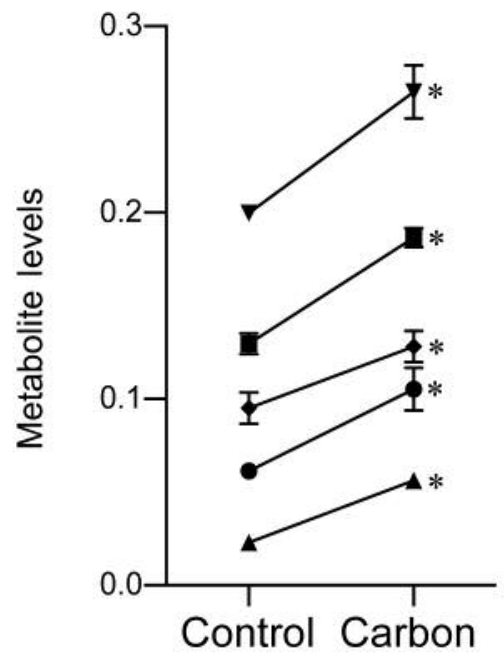

Figure 3. Carbon ions significantly altered the levels of several metabolites. (A) The top panel shows metabolites that were altered in five cell lines and $(B)$ the bottom panel shows metabolites that were altered in four cell lines. The asterisks indicate significance by Student's t-test with the Benjamini-Hochberg procedure.

showed that the TCA cycle was down-regulated two days after 1.1 or 4.5 Gy of X-ray irradiation in urine samples obtained from mice (18). Similarly, Pannkuk et al. showed that the TCA cycle was down-regulated one week after 2-10 Gy of Xray irradiation in urine and serum samples from non-human primates (19). These results are not consistent with our findings that the TCA cycle was up-regulated $24 \mathrm{~h}$ after $1 \mathrm{~Gy}$ of carbon ion or $3 \mathrm{~Gy}$ of X-ray irradiation. This discrepancy between the current study and previous studies may be due to the difference in radiation responses between cancer cells and normal cells and/or differences in the time of sampling and warrants further research.

2-HG is derived from $\alpha-\mathrm{KG}$, which is an intermediary metabolite of the TCA cycle, and is produced by the enzymes in the mitochondrial matrix and cytoplasm (17). 2$\mathrm{HG}$ exists as enantiomers, i.e., $\mathrm{D}(\mathrm{R})-2-\mathrm{HG}$ and $\mathrm{L}(\mathrm{S})-2-\mathrm{HG}$ 
A

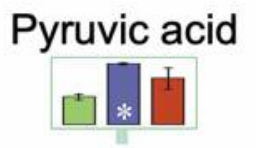

Acetyl coenzyme A

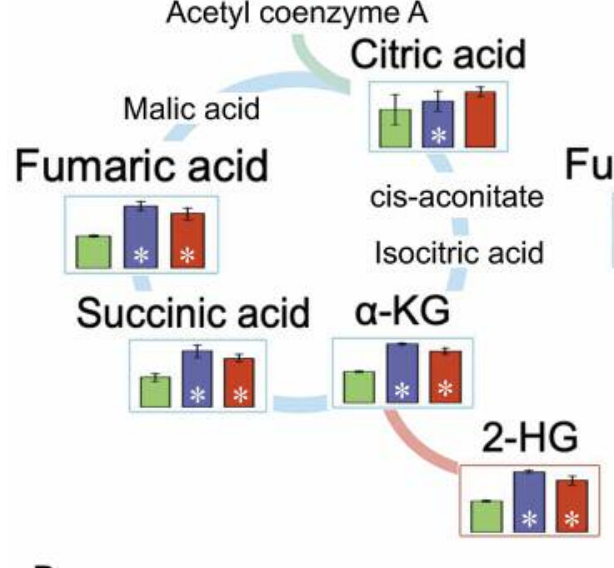

D

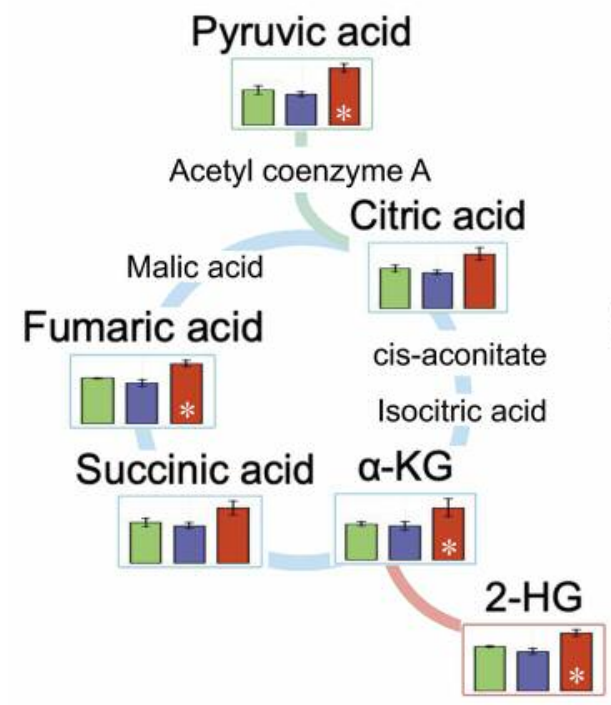

B

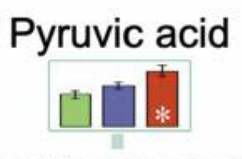

Acetyl coenzyme A

Citric acid

Malic acid

Fumaric acid

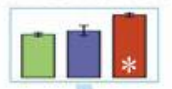

cis-aconitate

Isocitric acid

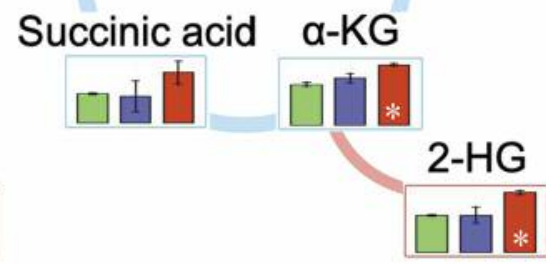

E

Pyruvic acid

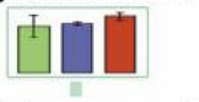

Acetyl coenzyme A
C

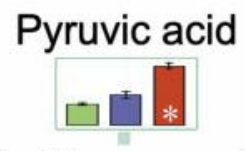

Acetyl coenzyme A

Citric acid

Malic acid

Fumaric acid

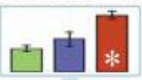

cis-aconitate

Isocitric acid

Succinic acid $\alpha-K G$

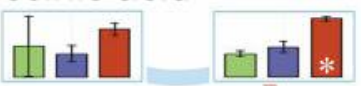

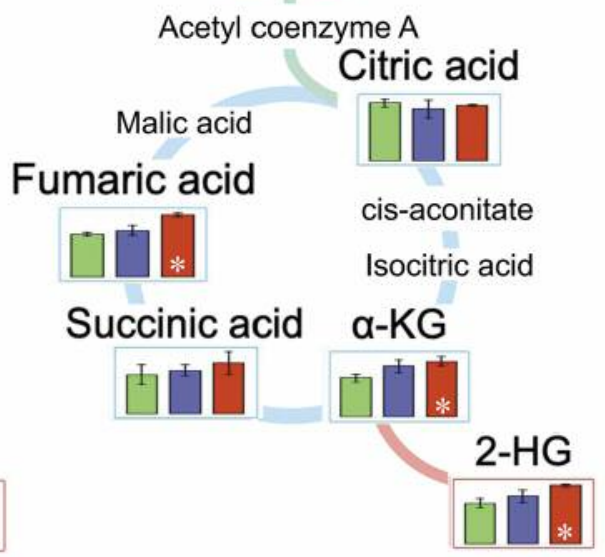

Figure 4. A metabolome data map of the tricarboxylic acid cycle in each cell line. Each bar represents the relative levels of metabolites in the control group (green bar), X-ray-treated group (blue bar) and carbon ion-treated group (red bar). Data are presented as mean \pm standard deviation. * $q$-values ( $p$-Values adjusted by Benjamini-Hochberg procedure) $<0.05$ compared to control. (A) A549, (B) MIA PaCa-2, (C) MeWo, (D) HuCCa1 and (E) U2OS cells.

(20). D-2-HG is an oncometabolite overproduced in cancers containing IDH1/2 mutations, such as glioma, myeloid leukemia, and chondrosarcoma. On the other hand, L-2-HG is efficiently produced by lactate dehydrogenase and malate dehydrogenase under hypoxia regardless of IDH status, and its steady-state levels are low. In this study, we found that carbon ions up-regulated 2-HG. Gelman et al. showed that cells with IDH $1 / 2$ mutations are more sensitive to oxidative stress induced by ionizing irradiation due to the consumption of NADPH associated with 2-HG synthesis (21). However, the relationship between ionizing irradiation and 2-HG synthesis is not fully elucidated. Further research to elucidate the mechanisms underlying the relationship between 2-HG synthesis and carbon ion irradiation, and to distinguish the enantiomer of 2-HG involved are needed.

The dose of irradiation used in this study was $1 \mathrm{~Gy}$ for carbon ions and $3 \mathrm{~Gy}$ for X-rays, based on the fact that the dose unit of Gy (relative biological effectiveness), calculated by 
multiplying the physical dose by three, is used in carbon ion radiotherapy (22). Nevertheless, the relative biological effectiveness is approximately 2.0 when in vitro clonogenic survival was used as an endpoint $(23,24)$. From this standpoint, 3 Gy of X-rays should produce at least an equivalent or greater cell-killing effect than $1 \mathrm{~Gy}$ of carbon ions. Despite this notion, our results showed that carbon ions induced greater metabolic alterations than X-rays. In general, the greater biological effect of carbon ions is associated with the induction of large and complex double-stranded breaks in DNA $(3,4)$. Therefore, the metabolic alterations observed in this study should be further studied in the context of DNA damage.

In this study, we treated cells with carbon ions using a clinically relevant beam setting, i.e., at the center of $6 \mathrm{~cm}$-width SOBP. In the clinic, carbon ion beams have various linear energy transfer (LET). The biological effectiveness of carbon ions depends on the LET (25). Besides, higher LET radiations cause more spatially heterogeneous ionization, leading to an increased proportion of 'un-hit' cells than when lower LET is used at the same physical dose $(3,26)$. Considering this issue, further research is needed to explore the relationship between metabolic alterations and the LET used.

One limitation of this study is that we could not analyze the metabolic changes of nucleic acid due to the characteristics of GC-MS analysis. Another limitation is that we did not analyze the molecular mechanisms underlying the metabolic alterations or the cell damage induced by carbon ions. These issues warrant further research.

In summary, we explored the metabolic alterations in various human cancer cells induced by carbon ions using clinically relevant beam settings. To the best of our knowledge, this is the first demonstration of up-regulation of TCA cycle intermediates and the downstream oncometabolite 2-HG following carbon ion irradiation. Further research is warranted to investigate the underlying molecular mechanisms, including those associated with DNA damage responses.

\section{Supplementary Material}

Supplementary data and figures are provided in the following link: https://www.researchgate.net/profile/Naoto-Osu/publications

\section{Conflicts of Interest}

All Authors declare no conflicts of interest.

\section{Authors' Contributions}

N.O. performed experiments, analyzed data, wrote the manuscript and acquired funding. H.M. performed experiments, wrote the manuscript and supervised the project. T. Oike analyzed data and reviewed the manuscript. T. Ohno supervised the entire project and acquired funding. All Authors have read and agreed to the publication of the manuscript.

\section{Acknowledgements}

We thank Ms. Ami Maruyama of Human Metabolome Technologies for technical assistance. This work was supported by grants-in-aid from the Ministry of Education, Culture, Sports, Science, and Technology of Japan for programs for Leading Graduate Schools, Cultivating Global Leaders in Heavy Ion Therapeutics and Engineering. This work was also supported by the Gunma University Heavy Ion Medical Center.

\section{References}

1 Schlaff CD, Krauze A, Belard A, O'Connell JJ and Camphausen KA: Bringing the heavy: carbon ion therapy in the radiobiological and clinical context. Radiat Oncol 9(1): 88, 2014. PMID: 24679134. DOI: 10.1186/1748-717X-9-88

2 Tinganelli W and Durante M: Carbon ion radiobiology. Cancers (Basel) 12(10): 3022, 2020. PMID: 33080914. DOI: 10.3390/ cancers 12103022

3 Hagiwara Y, Niimi A, Isono M, Yamauchi M, Yasuhara T, Limsirichaikul S, Oike T, Sato H, Held KD, Nakano T and Shibata A: 3D-structured illumination microscopy reveals clustered DNA double-strand break formation in widespread $\gamma \mathrm{H} 2 \mathrm{AX}$ foci after high LET heavy-ion particle radiation. Oncotarget 8(65): 109370-109381, 2017. PMID: 29312614. DOI: $10.18632 /$ oncotarget.22679

4 Kobayashi D, Oike T, Shibata A, Niimi A, Kubota Y, Sakai M, Amornwhichet N, Yoshimoto Y, Hagiwara Y, Kimura Y, Hirota Y, Sato H, Isono M, Yoshida Y, Kohno T, Ohno T and Nakano T: Mitotic catastrophe is a putative mechanism underlying the weak correlation between sensitivity to carbon ions and cisplatin. Sci Rep 7: 40588, 2017. PMID: 28091564. DOI: 10.1038/srep40588

5 Liu X and Locasale JW: Metabolomics: A primer. Trends Biochem Sci 42(4): 274-284, 2017. PMID: 28196646. DOI: 10.1016/j.tibs.2017.01.004

6 Vander Heiden MG, Cantley LC and Thompson CB: Understanding the Warburg effect: the metabolic requirements of cell proliferation. Science 324(5930): 1029-1033, 2009. PMID: 19460998. DOI: 10.1126/science.1160809

7 Xiang K, Jendrossek V and Matschke J: Oncometabolites and the response to radiotherapy. Radiat Oncol 15(1): 197, 2020. PMID: 32799884. DOI: 10.1186/s13014-020-01638-9

8 Patel D, Thompson MD, Manna SK, Krausz KW, Zhang L, Nilubol N, Gonzalez FJ and Kebebew E: Unique and novel urinary metabolomic features in malignant versus benign adrenal neoplasms. Clin Cancer Res 23(17): 5302-5310, 2017. PMID: 28450405. DOI: 10.1158/1078-0432.CCR-16-3156

9 Oike T, Hirota Y, Dewi Maulany Darwis N, Shibata A and Ohno T: Comparison of clonogenic survival data obtained by pre- and post-irradiation methods. J Pers Med 10(4): 171, 2020. PMID: 33076277. DOI: 10.3390/jpm10040171

10 Takeo E, Sasano R, Shimma S, Bamba T and Fukusaki E: Solidphase analytical derivatization for gas-chromatography-massspectrometry-based metabolomics. J Biosci Bioeng 124(6): 700706, 2017. PMID: 28800906. DOI: 10.1016/j.jbiosc.2017.07.006

11 Nishiumi S, Kobayashi T, Kawana S, Unno Y, Sakai T, Okamoto K, Yamada Y, Sudo K, Yamaji T, Saito Y, Kanemitsu Y, Okita NT, Saito H, Tsugane S, Azuma T, Ojima N and Yoshida M: Investigations in the possibility of early detection of colorectal 
cancer by gas chromatography/triple-quadrupole mass spectrometry. Oncotarget 8(10): 17115-17126, 2017. PMID: 28179577. DOI: 10.18632/oncotarget.15081

12 Pang Z, Chong J, Zhou G, de Lima Morais DA, Chang L, Barrette M, Gauthier C, Jacques PÉ, Li S and Xia J: MetaboAnalyst 5.0: narrowing the gap between raw spectra and functional insights. Nucleic Acids Res 49(W1): W388-W396, 2021. PMID: 34019663. DOI: 10.1093/nar/gkab382

13 Junker BH, Klukas C and Schreiber F: VANTED: a system for advanced data analysis and visualization in the context of biological networks. BMC Bioinformatics 7: 109, 2006. PMID: 16519817. DOI: $10.1186 / 1471-2105-7-109$

14 Lin SH, Fan J, Zhu J, Zhao YS, Wang CJ, Zhang M and Xu F: Exploring plasma metabolomic changes in sepsis: a clinical matching study based on gas chromatography-mass spectrometry. Ann Transl Med 8(23): 1568, 2020. PMID: 33437767. DOI: $10.21037 / \mathrm{atm}-20-3562$

15 den Hartog I, Zwep LB, Vestjens SMT, Harms AC, Voorn GP, de Lange DW, Bos WJW, Hankemeier T, van de Garde EMW and van Hasselt JGC: Metabolomic profiling of microbial disease etiology in community-acquired pneumonia. PLoS One 16(6): e0252378, 2021. PMID: 34086721. DOI: 10.1371/journal.pone.0252378

16 Benjamini Y and Hochberg Y: Controlling the false-discovery rate: a practical and powerful approach to multiple testing. J Roy Statist Soc Ser B 57: 289-300, 1995.

17 Martínez-Reyes I and Chandel NS: Mitochondrial TCA cycle metabolites control physiology and disease. Nat Commun 11(1): 102, 2020. PMID: 31900386. DOI: 10.1038/s41467-019-13668-3

18 Goudarzi M, Mak TD, Chen C, Smilenov LB, Brenner DJ and Fornace AJ: The effect of low dose rate on metabolomic response to radiation in mice. Radiat Environ Biophys 53(4): 645-657, 2014. PMID: 25047638. DOI: 10.1007/s00411-0140558-1

19 Pannkuk EL, Laiakis EC, Authier S, Wong K and Fornace AJ Jr: Gas chromatography/mass spectrometry metabolomics of urine and serum from nonhuman primates exposed to ionizing radiation: impacts on the tricarboxylic acid cycle and protein metabolism. J Proteome Res 16(5): 2091-2100, 2017. PMID: 28351153. DOI: $10.1021 /$ acs.jproteome.7b00064

20 Intlekofer AM, Dematteo RG, Venneti S, Finley LW, Lu C, Judkins AR, Rustenburg AS, Grinaway PB, Chodera JD, Cross JR and Thompson CB: Hypoxia induces production of L-2hydroxyglutarate. Cell Metab 22(2): 304-311, 2015. PMID: 26212717. DOI: 10.1016/j.cmet.2015.06.023
21 Gelman SJ, Naser F, Mahieu NG, McKenzie LD, Dunn GP, Chheda MG and Patti GJ: Consumption of NADPH for 2-HG synthesis increases pentose phosphate pathway flux and sensitizes cells to oxidative stress. Cell Rep 22(2): 512-522, 2018. PMID: 29320744. DOI: 10.1016/j.celrep.2017.12.050

22 Kanai T, Endo M, Minohara S, Miyahara N, Koyama-ito H, Tomura H, Matsufuji N, Futami Y, Fukumura A, Hiraoka T, Furusawa Y, Ando K, Suzuki M, Soga F and Kawachi K: Biophysical characteristics of HIMAC clinical irradiation system for heavy-ion radiation therapy. Int J Radiat Oncol Biol Phys 44(1): 201-210, 1999. PMID: 10219815. DOI: 10.1016/s03603016(98)00544-6

23 Amornwichet N, Oike T, Shibata A, Nirodi CS, Ogiwara H, Makino H, Kimura Y, Hirota Y, Isono M, Yoshida Y, Ohno T, Kohno T and Nakano T: The EGFR mutation status affects the relative biological effectiveness of carbon-ion beams in nonsmall cell lung carcinoma cells. Sci Rep 5: 11305, 2015. PMID: 26065573. DOI: $10.1038 /$ srep 11305

24 Osu N, Kobayashi D, Shirai K, Musha A, Sato H, Hirota Y, Shibata A, Oike T and Ohno T: Relative biological effectiveness of carbon ions for head-and-neck squamous cell carcinomas according to human papillomavirus status. J Pers Med 10(3): 71, 2020. PMID: 32722522. DOI: 10.3390/jpm 10030071

25 Takahashi A, Kubo M, Ma H, Nakagawa A, Yoshida Y, Isono M, Kanai T, Ohno T, Furusawa Y, Funayama T, Kobayashi Y and Nakano T: Nonhomologous end-joining repair plays a more important role than homologous recombination repair in defining radiosensitivity after exposure to high-LET radiation. Radiat Res 182(3): 338-344, 2014. PMID: 25117625. DOI: 10.1667/ RR13782.1

26 Nakajima NI, Brunton H, Watanabe R, Shrikhande A, Hirayama R, Matsufuji N, Fujimori A, Murakami T, Okayasu R, Jeggo P and Shibata A: Visualisation of $\gamma \mathrm{H} 2 \mathrm{AX}$ foci caused by heavy ion particle traversal; distinction between core track versus non-track damage. PLoS One 8(8): e70107, 2013. PMID: 23967070. DOI: 10.1371/journal.pone.0070107
Received September 18, 2021

Revised October 30, 2021

Accepted November 2, 2021 